\title{
Influence of edge effects on the load transmitted by adhesively-bonded assemblies
}

\author{
R. Créac'hcadec ${ }^{1,2}$, L. Sohier ${ }^{1,3}$, J. Y. Cognard ${ }^{1,2}$ \& B. Gineste ${ }^{1,3}$ \\ ${ }^{1}$ Laboratoire Brestois de Mécanique et des Systèmes, France \\ ${ }^{2}$ ENSIETA, France \\ ${ }^{3}$ Université de Brest, France
}

\begin{abstract}
This paper presents a contribution to the influence of edge effects on the load transmitted by adhesively-bonded assemblies. Bonded assemblies are mostly characterized by the presence of stress concentrations at the bottom of the adhesive and in its thickness near the interface. This phenomenon, called edge effects, plays a very important role in the macroscopic observed behaviour of an adhesive in an assembly. Thus, to describe its behaviour it is necessary to propose solutions to reduce the edge effects for the bonded test specimens used to characterize the adhesive and for industrial applications. Mastering edge effects improves the efficiency of bonded parts and reduces the dispersions of the loads at failure. In the first part a modified Arcan test fixture is described to analyze the behaviour of an adhesive in an assembly under traction-shear loadings. The test specimens use a specific geometry to reduce the edge effects (beaks). In the second part, edge effects are analyzed by the finite element method under elastic hypothesis. In the third part, experimental results for traction-shear Arcan tests are presented. And finally, this method to reduce edge effects is applied to the bonding of a rail on a composite mast for high competitive sailing boats.
\end{abstract}

Keywords: adhesive joint, edge effects, finite element analysis, experimental results, joint design.

\section{Introduction}

This study is concerned with increasing the performance of adhesively bonded assemblies which offer many advantages. But the experimental and numerical 
analyses of the mechanical behaviour of bonded joints are particularly made difficult by the edge effects. Stress singularities can contribute to the initiation and the propagation of cracks in adhesive joints $[1,2]$. Therefore, understanding the stress distribution in an adhesive joint can lead to improvements and to develop reliable experimental tests in order to analyse the non-linear behaviour of an adhesive in an assembly. Several analytical studies have been proposed to analyse the influence of the geometry on the stress singularities for bi-material joints [3]. But, precise finite element computations are useful to analyse the stress distributions in order to optimise the design of bonded assemblies taking into account the influence of the various parameters such as: the geometry of the bonded assembly, the interactions between the two interfaces of the thin joint and the non linear behaviour of the adhesive. In order to complete the numerical study, experimental results, using a modified Arcan device [4], under tensileshear loadings are proposed for two geometries (a usual geometry with quite large stress concentrations, and a geometry designed to strongly limit the influence of edge effects).The same strategy is used to analyse the mechanical behaviour of an industrial type bonded assembly under complex loading (tension, shear, bending) using a mini-structure. Experimental results underline that geometries which reduce the influence of edge effects are associated with an increase of the transmitted load and a decrease of the scatter in the results. This study makes it possible to optimize the dimensioning of adhesively-bonded assemblies.

\section{Modified Arcan experimental fixture}

\subsection{Description of the experimental device}

In order to be able to study the behavior of the adhesive as a function of the normal stress component, a modified Arcan fixture has been developed, which enables compression or tension to be combined with shear loads (Figure 1). Numerical simulations in linear elasticity, for bi-material structures show that the use of a special geometry for the substrate (a beak close to the adhesive joint) makes it possible to eliminate the contribution of the singularities due to edge effects [5]. One wants to obtain the most uniform possible stress field in the adhesive joint and maximum stress in the centre in order to limit the influence of defects. Moreover non linear simulations taking into account the fixing system of the substrates on the supporting fixture were used to optimize the design of the complete fixture in order to prevent pre-loading of the adhesive joint [6]. To preserve the simplicity associated with the Arcan fixture, and the use of a traditional tensile testing machine, a specimen with rectangular section was proposed taking into account the problems involved in machining. This experimental fixture associated with non-contact extensometry and optimization techniques allows us to analyze, for radial loadings, the non linear behavior of an adhesive joint (epoxy resin Huntsman ${ }^{\mathrm{TM}}$ Araldite $^{\mathrm{B}} 420 \mathrm{~A} / \mathrm{B}$ has been used [7]). 


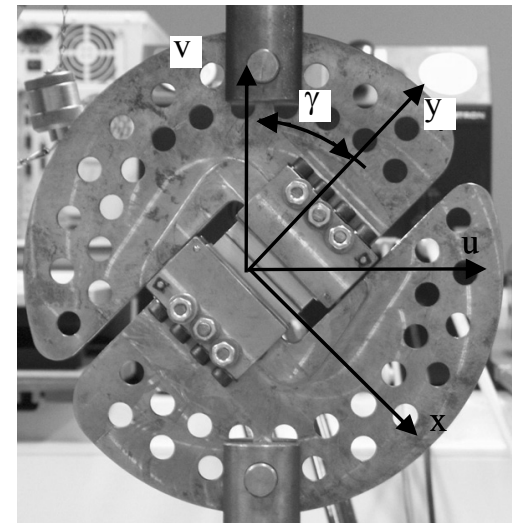

(a) tensile-shear test

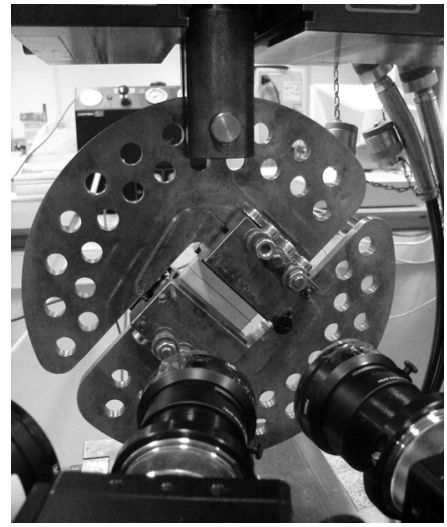

(b) Non-contact extensometry System [8]

Figure 1: $\quad$ The modified Arcan fixture.

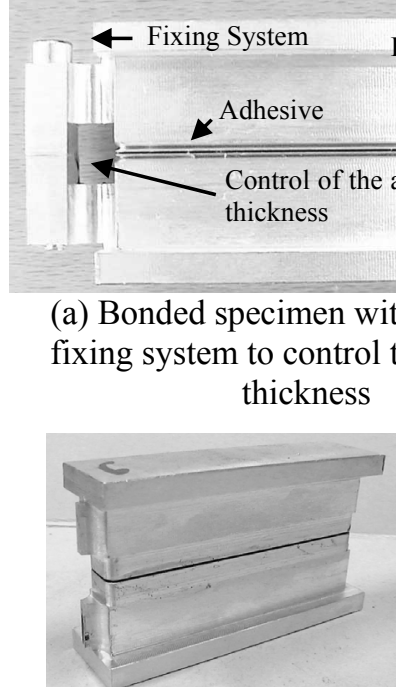

(c) Arcan test specimen just before testing

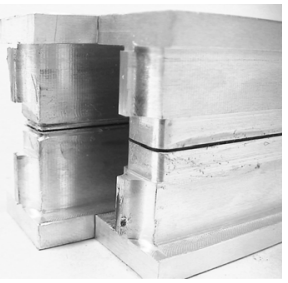

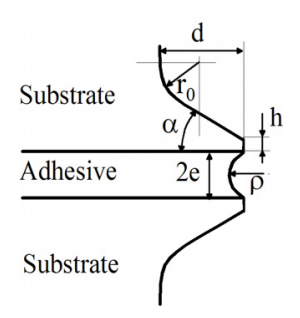

b) Zoom of the beaks and its geometry

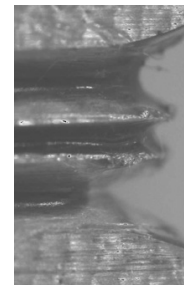

(d) beaks

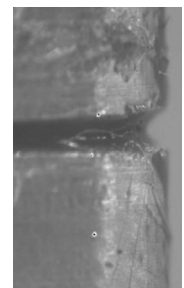

(e) straight edges

Figure 2: $\quad$ Arcan test specimen with beaks and straight edges. 


\subsection{Description of the Arcan test specimen}

The design of this modified Arcan fixture (use of beaks, cleaning of the free edges of the adhesive, fixing system) allows to strongly limit the edge effects and to prevent pre-loading of the adhesive (Figure 1) [9]. For this test a bonded specimen with rectangular section $\left(9.5 \times 65 \mathrm{~mm}^{2}\right)$ was proposed. The main parameters which define the geometry of the bonded specimen are such that: $\mathrm{e}=$ $0.2 \mathrm{~mm}, \mathrm{~h}=0.2 \mathrm{~mm}, \mathrm{~d}=0.5 \mathrm{~mm}, r_{0}=0.8 \mathrm{~mm}, \rho=1.5 \mathrm{e}, \alpha=45^{\circ}$ (Figure 2(b)).

The test specimen described in the figure $2 \mathrm{a}$, presents the geometry of the two substrates and the adhesive. The thickness of the adhesive is controlled using two fixing systems i.e. the adhesive thickness is $0.4 \mathrm{~mm}$. They are also used to control the position of the two substrates and to reduce the geometric dispersions linked to the bonding operation. After curing the adhesive, the fixing system is cut very simply. Figure 2(c) presents the bonded specimens just before testing. The beaks and the straight substrates with cleaned edges are described in figures $2(\mathrm{~d})$ and $2(\mathrm{e})$.

\section{Numerical analysis of the edge effects for tensile-shear tests}

The behaviour of the adhesive (epoxy resin Huntsman ${ }^{\mathrm{TM}}$ Araldite $^{\circledR} 420 \mathrm{~A} / \mathrm{B}$ ) is assumed to be isotropic ( $E_{a}=2.0 \mathrm{GPa}, v_{a}=0.3$ ) and the elastic limit was identified for 2D tensile-shear loadings [10], and proposed in the following form:

$$
\frac{\sigma_{y y}^{2}}{R_{a}^{2}}+\frac{\sigma_{x y}^{2}}{S_{a}^{2}}=1
$$

with $R_{a}=40 \mathrm{MPa}$ and $S_{a}=25 \mathrm{MPa}[10]$.

Figures 3 and 4 present the evolution of the peel stress (yy stress), of the shear stress (xy stress) and of the equivalent stress with respect to the $x$ axis $(\mathrm{x} \in[-$ $32.5 ; 32.5 \mathrm{~mm}$ ], Figure 2a) through the adhesive thickness in the case of a tensile-shear loading $\left(\gamma=45^{\circ}\right.$, Figure 1). In Figures 3 and $4, y=0$ corresponds to the middle plane of the adhesive and $\mathrm{y}=\mathrm{e}$ corresponds to the interface. To facilitate the analysis of the results, only the minimum and the maximum values are represented. For those 2D simulations, substrates with straight edges and with beaks have been used with straight edges of the adhesive $(\rho=\infty)$ and with cleaned edges $(\rho=1.5 e)$. Those results underline the beneficial effects of the beaks and of the cleaning of the free edges of the adhesive.

It can be seen that, for cleaned adhesive edges, with straight substrates or with substrates with beaks the maximum equivalent stress is not obtained in close to the free edges of the adhesive. Moreover, for cleaned adhesive edges, the maximum stress state close the free edge is inside the joint and not close to the adhesive-substrate interface (which is often the weaker part of the joint) as for straight adhesive edges. 
yy stress

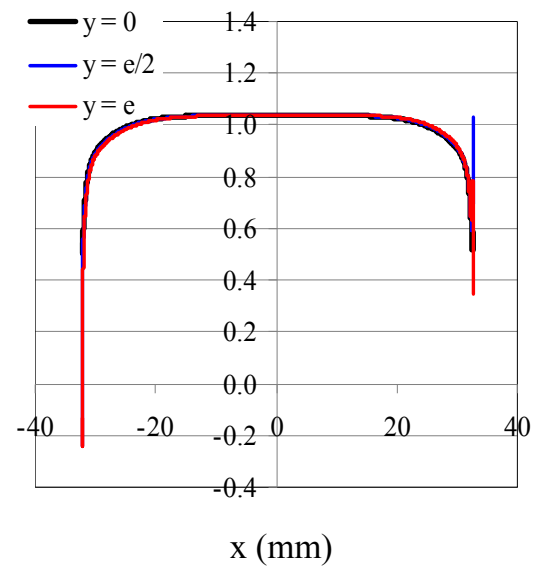

(a) cleaned adhesive edges

equivalent stress

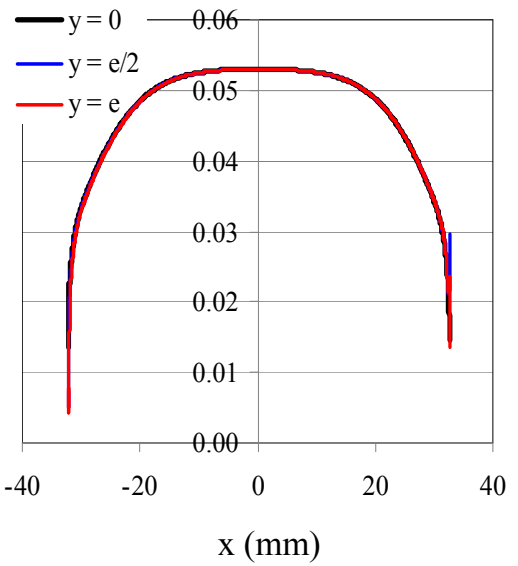

(c) cleaned adhesive edges xy stress

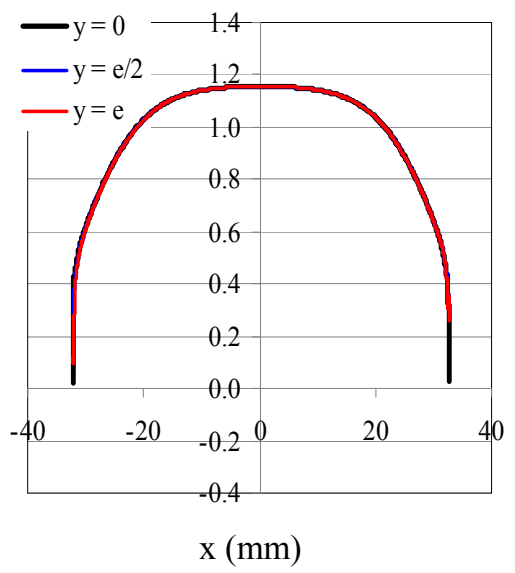

(b) cleaned adhesive edges

equivalent stress

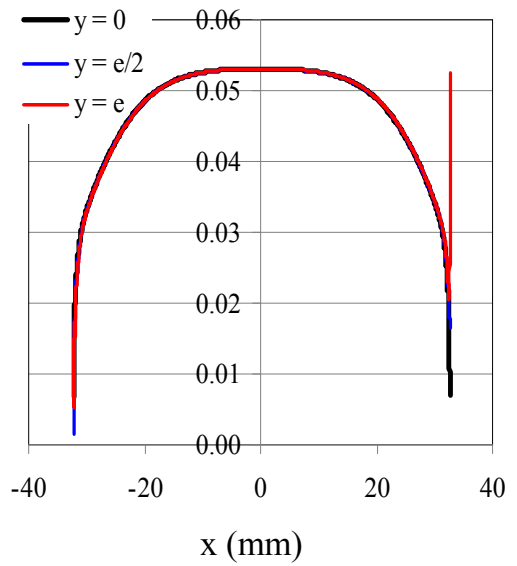

(d) straight adhesive edges

Figure 3: $\quad$ Stress distribution along the overlap for substrates with beaks. 
yy stress

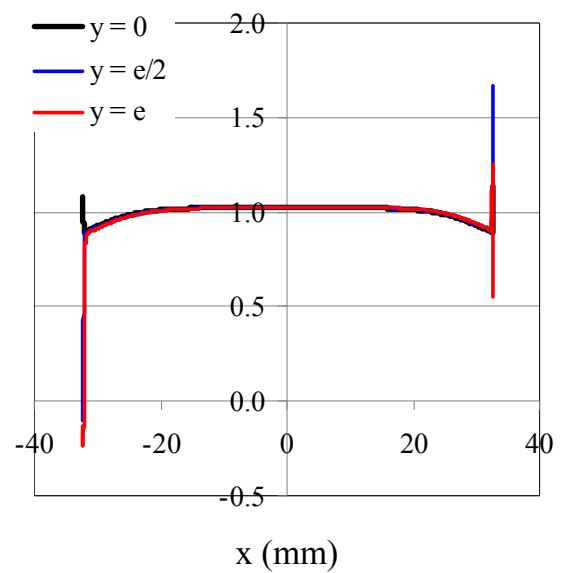

(a) cleaned adhesive edges

equivalent stress

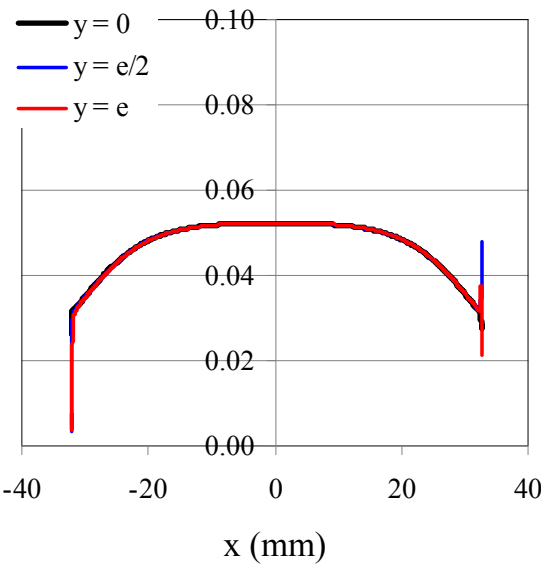

(a) cleaned adhesive edges xy stress

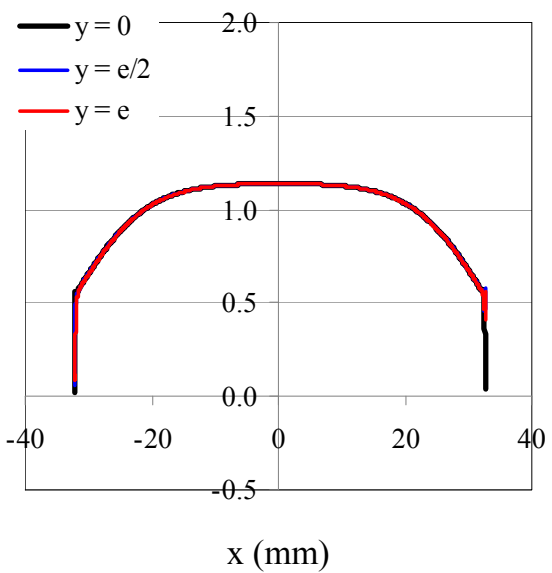

(b) cleaned adhesive edges

equivalent stress

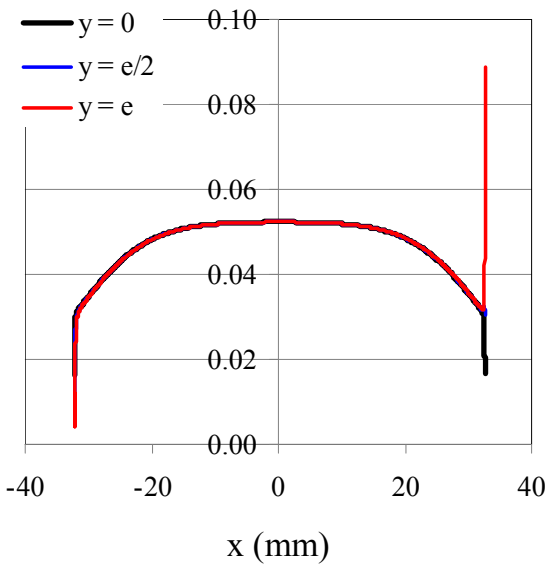

(b) straight adhesive edges

Figure 4: $\quad$ Stress distribution along the overlap with straight substrates.

\section{Experimental results for a tensile-shear test}

\subsection{Arcan test specimen used}

Two specific geometries for the substrates have been used in order to reduce the edge effects. Figures 2(c)-(e) present two specimens just before testing. Figure 2(d) describes the geometry of the beak with cleaning all around the 
adhesive. Figure 2(e) presents straight edges with cleaning. The thickness of the adhesive is controlled and measured at $0.4 \mathrm{~mm}+/-0.02 \mathrm{~mm}$.

\subsection{Experimental load-displacement diagrams}

To analyze the influence of the edge effects on the behaviour of the adhesive and on the dispersions, several traction-shear tests were made with the two geometries presented in the figure 2. Figure 5 presents the results in the loaddisplacement diagram in the tangential direction (FT, DT) and in the normal direction (FN, DN) for an angle $\left(\gamma=45^{\circ}\right.$, Figure 1a) and a load displacement rate of $0.5 \mathrm{~mm} / \mathrm{min}$. F is the applied load. DT and DN are respectively the relative displacements of both ends of the adhesive in the tangential and respectively in the normal directions. A very low scatter in the results can be noted for these two geometries. The load at failure is a little bit higher for the geometry with beaks. Moreover, the useful section with beaks is a little smaller tan for straight adhesive edges. It is important to notice that the mechanical surface preparation lead to a little increase of the thickness of the adhesive close to the free edges, which leads to a reduction of the stress concentrations [5].
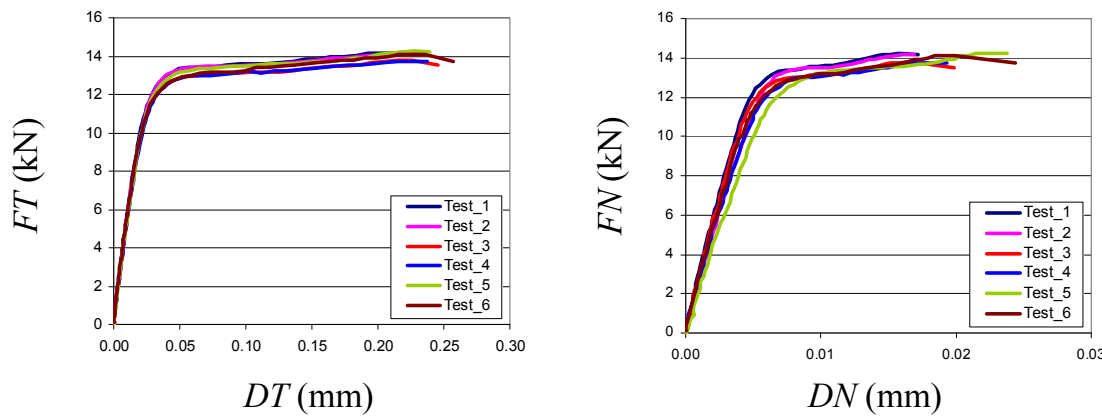

(a) Substrates with beaks and cleaning
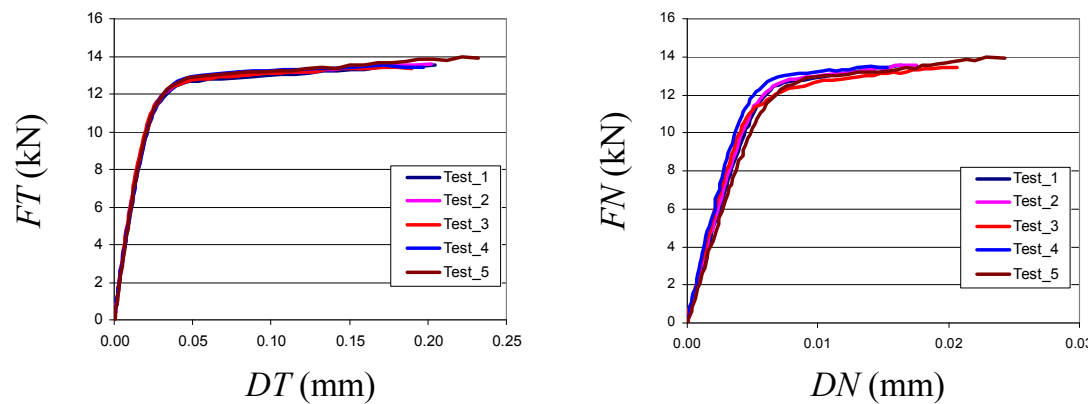

(b) Substrates with straight edges and cleaning

Figure 5: Load-displacement diagrams in the tangential and normal directions for tensile-shear tests. 
These results underline that it is important to design bonded specimens with the maximum stress state not close to the free edges of the joint. The stress analysis has to take into account the stress through the adhesive thickness in order to have the influence of the edge effects [9]. In fact, the important point, for computations under linear elastic assumption, is to limit the maximal equivalent stress close to the free edges of the adhesive in order to limit the influence of defects, even if stress concentrations can exist.

\section{Bonding of a rail on a composite mast for high competitive sailing boat}

\subsection{Presentation of the problem}

In order to analyze the influence of various geometrical parameters on industrial type applications, a test to characterize the bonding of a rail (used to guide the mainsail) to a composite mast on a racing yacht has been developed. The first objective of this test is to analyze and optimize the behaviour of this type of hybrid bonded assembly; it is necessary to study the influence of various solutions making it possible to limit the edge effects for this application and to check the delamination resistance of the mast knowing that the mast is not usually designed for such constraints, because of the presence of attaching bolts. A detailed analysis of this test requires comparisons between experimental and numerical results; it is an interesting example to validate the numerical tool which is under development in order to optimise adhesively-bonded structures [10].

The analysis of various configurations requires the development of a relatively simple experimental device. Thus, to carry out this test, we have fixed the back face of a mast of a 60 foot IMOCA racing yacht onto a modular support allowing us to load the rail in various configurations using a tensile testing machine (Figure 6). During the design of this fixture, different technical

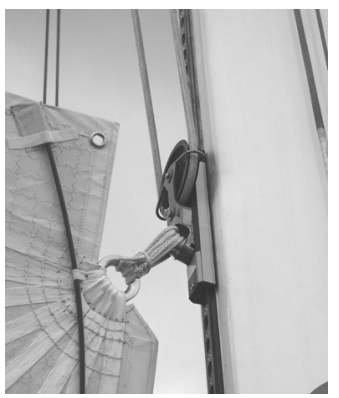

(a) Industrial problem

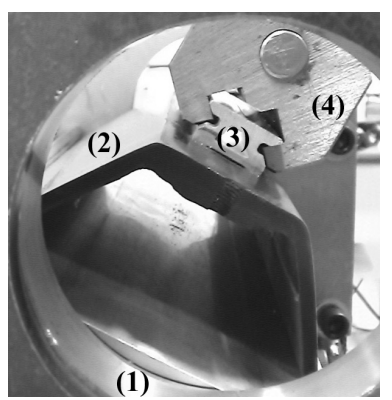

(b) Experimental fixture 1 - support

$$
3 \text { - rail } 4 \text { - trolley }
$$

Figure 6: Presentation of the experimental device. 
solutions have been used to limit the parasite effects; moreover improvements are under development. In order to obtain a test representative of the real problem, starting from a piece of mast, various Finite Element computations were carried out.

\subsection{Test on a mini-structure: optimisation of the design of the geometry of the different parts}

In order to analyse the influence of the edge effects on the behaviour of the assembly studied here, a modification of the experimental device has been proposed. The idea is to limit the difficulties; as we are analysing geometric effects we have replaced the composite mast part ((2) on figure 6b) by an intermediate aluminium support $\left(\left(2^{\prime}\right)\right.$ on figure $\left.6 \mathrm{~b}\right)$. Precise positioning of the different parts is obtained by the use of a special fixture during bonding, figure 7 . Four different geometries have been tested. Geometry "a" is characterised with straight edges of the rail and of the support. For geometry "b" a larger intermediate support is used with straight edges. Geometry "c" uses sharp beaks on the two parts to limit the influence of edge effects. For geometry "d", a small increase in the thickness of the joint is proposed to limit the influence of edge effects. For the different tests, the edge of the adhesive is cleaned in order to reduce the edge effects.

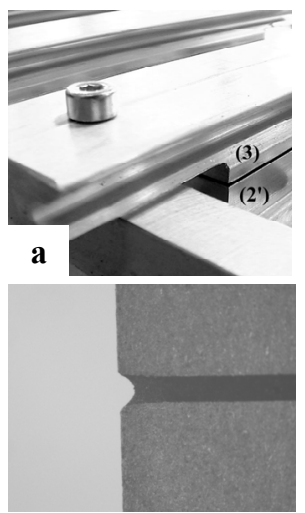

(b) Geometry "a"

Straight cleaned edges
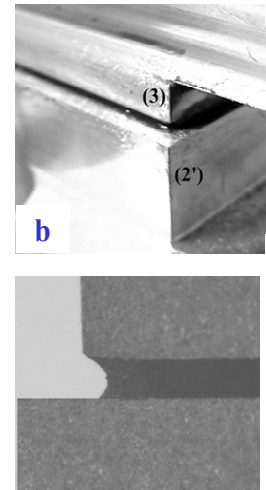

(c) Geometry "b" massive cleaned edges
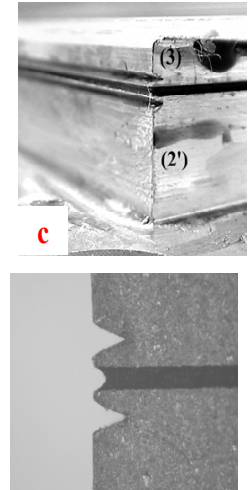

(d) Geometry "c" cleaned beaks
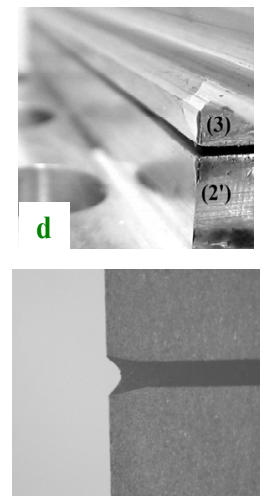

(e) Geometry "d" chamfered cleaned beaks

Figure 7: Presentation of the experimental device with intermediate support.

For the real application with hybrid bonded assemblies the loading is characterized with tension-shear and bending; the influence of edge effects are different to those for Arcan tests with only tension-shear loadings. Therefore such assemblies require a specific study.

Figure 8 presents the transmitted load (at failure) for first tests with a part of the composite mast (figure 6) and for the different geometries with an 


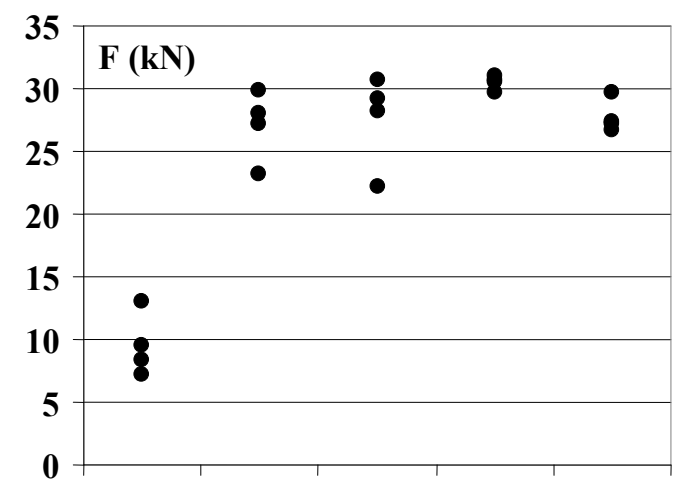

Comp G "a" G "b" G "c" G "d"

Figure 8: Load at failure for the different geometries proposed.

intermediate aluminium support (figure 7). For each geometry, four tests have been made in order to analyze the result variability. Significant differences are obtained for the different configurations. Numerical simulations have shown that the edge effects are lower for geometries "c" and " $d$ " than for geometries "a" and "b". Moreover it is important to note that experimental results are less scattered with strategies which strongly limit the edge effects. The cleaning is more difficult to manage for geometry "d" than for geometry "c", which may explain some differences between the two solutions.

The first tests to characterize this industrial type hybrid bonded assembly were made directly on part of the mast of a racing yacht with part of a rail. However, this approach is complex, as the different parts of this assembly have not been designed for using only an adhesive joint: the classical assembly uses an adhesive joint and bolts; thus the parameters of this assembly have to be analyzed in order to optimize the transmitted loading.

\section{Conclusion}

Analyzing the behaviour of an adhesive in an assembly requires bonded test specimens with lower stress concentrations. Edge effects play a very important role in the mechanical behaviour observed. Thanks to specific geometries like the beaks developed in this paper and the cleaning of the edges of the adhesive it is possible to reduce significantly the stresses at each bottom of the overlap and in the thickness of the adhesive. Experimental tests and numerical analyses are in good match to underline that it is important to design bonded specimens with the maximum stress state not close to the free edges of the joint. For traction-shear tests the reliability of the modified Arcan test specimen is very well thanks to the master of the edge effects with beaks and cleaning. From a numerical point of view, considering elastic assumption, edge effects are very reduce with the geometry and preserve the interface, often the weaker part of a bonded assembly, 
from the maximum stress state. These results can be adapted to industrial applications with a very complex stress state in the adhesive, such as the bonding of a rail on a mast. The local geometry of the free edge plays an important role, not on the level of the maximum load at failure, but significantly on the dispersion of the results. Applying a design criterion to industrial applications with this geometry thanks to the reduction of the dispersions can optimize the bonded assembly.

\section{References}

[1] Adams, R.D. Adhesive Bonding: Science, Technology and Applications, Woodhead Publishing Ltd. (England), 2005.

[2] Da Silva, L. F. M. \& Ochsner, A., Modeling Of Adhesively Bonded Joints, Springer (England), 2008.

[3] Leguillon, D., Laurencin, J. \& Dupeux, M., Failure initiation in an epoxy joint between two steel plates. European Journal of Mechanics A/Solids, 22, pp 509-524, 2003.

[4] Cognard, J.Y., Créac'hcadec, R., Davies, P. \& Sohier, L., Numerical modelling of the non-linear behavior of adhesively-bonded assemblies, Innovation In Engineering Computational Structures Technology, SaxeCoburg Publications, ISBN 1-874672-27-X, 11, pp 225-247, 2006.

[5] Cognard, J.Y., Numerical analysis of edge effects in adhesively-bonded assemblies. Application to the determination of the adhesive behaviour. Computers \& Structures, 86, pp 1704-1717, 2008.

[6] Cognard, J. Y., Davies, P., Sohier, L. \& Créac'hcadec, R., A study of the non-linear behaviour of adhesively-bonded composite assemblies. Composites Structures, 76, pp 34-46, 2006.

[7] Huntsman ${ }^{\mathrm{TM}}$ Structural Adhesives - Araldite $^{\circledR} 420$ A/B, Publication No A 161, UK, 2004.

[8] GOM Optical Measuring Techniques: Aramis 3D deformation analysis RisOrangis, France, www.gom.com.

[9] Cognard, J.Y., Créac'hcadec, R., Sohier, L. \& Davies, P., Analysis of the non linear behaviour of adhesives in bonded assemblies. Comparison of TAST and ARCAN tests. International Journal of Adhesion \& Adhesives, 28, pp 393-404, 2008.

[10] Créac'hcadec, R., Cognard, J.Y. \& Heuzé, Th., On modelling the behaviour of thin adhesive films in bonded assemblies with interface elements, Journal of Adhesion Science and Technology, 22, pp 1541-1563, 2008. 